\title{
The properties of functional inclusions and Hyers-Ulam stability
}

\author{
MAGDALENA PisZCZEK
}

\begin{abstract}
We prove that a set-valued function satisfying some functional inclusions admits, in appropriate conditions, a unique selection satisfying the corresponding functional equation. As a consequence we obtain the result on the Hyers-Ulam stability of that functional equation.
\end{abstract}

Mathematics Subject Classification (2010). 39B05, 39B82, 54C60, 54C65.

Keywords. Stability of functional equation, set-valued map, selection.

The first result on the stability of functional equations was given in 1941 by Hyers [7] who proved the following theorem:

Let $X$ be a linear normed space, $Y$ a Banach space and $\epsilon>0$. Then for every function $f: X \rightarrow Y$ satisfying the inequality

$$
\|f(x+y)-f(x)-f(y)\| \leq \epsilon, \quad x, y \in X
$$

there exists a unique additive function $g: X \rightarrow Y$ such that

$$
\|f(x)-g(x)\| \leq \epsilon, \quad x \in X .
$$

This was a first answer given to a question proposed by S.M. Ulam in a talk at a conference at the Wisconsin University in 1940 and it represents the starting point of the Hyers-Ulam stability theory of functional equations (see $[8,9]$ ). The subject was later strongly developed by many authors, see for example: $[1,4,11,12,16]$. An interesting connection between the stability of the Cauchy equation and subadditive set-valued functions was established by Smajdor [17] and Gajda and Ger [6]. They observed that if $f$ satisfies (1), then the set-valued function $F: X \rightarrow n(Y)(n(Y)$ denotes the family of all nonempty subsets of $Y$ ) given by

$$
F(x)=f(x)+\bar{B}(0, \epsilon), \quad x \in X,
$$

where $\bar{B}(0, \epsilon)$ is the closed ball of center 0 and radius $\epsilon$, is subadditive (i.e., $F(x+y) \subset F(x)+F(y), x, y \in X)$ and the function $g$ from relation (2) is 
an additive selection of $F$ (i.e., $g(x+y)=g(x)+g(y)$ and $g(x) \in F(x)$ for $x, y \in X)$.

The question that appeared as a consequence was: under what conditions a subadditive set-valued map admits an additive selection. An answer to this equation can be found in [6]. Next, the previous result was extended by Nikodem and Popa to set-valued functions satisfying general linear inclusions:

$$
\begin{aligned}
& F(\alpha x+\beta y+c) \subset \gamma F(x)+\delta F(y)+C, \\
& \alpha F(x)+\beta F(y) \subset F(\gamma x+\delta y+c)+C,
\end{aligned}
$$

where $\alpha, \beta, \gamma, \delta \in \mathbb{R}, X$ is a real vector space, $Y$ is a real Banach space, $F: X \rightarrow$ $n(Y), c \in X, C \in 2^{Y}$ (see $\left.[10,13-15]\right)$.

It is interesting that we can prove the stability of functional equations corresponding to the functional inclusions considered. This is a motivation to study at first some inclusions (cf. [3]).

Through this paper we assume that $K$ is a nonempty set, $Y$ is a normed space over a field $\mathbb{K} \in\{\mathbb{R}, \mathbb{C}\}$ and $h$ is the Hausdorff distance derived from the norm in $Y$. We will denote by $b d(Y)$ the family of all nonempty and bounded subsets of $Y$ and $b c l(Y)$ stands for the family of all closed sets of $b d(Y)$. The number $\delta(A)=\sup \{\|x-y\|: x, y \in A\}$ is said to be the diameter of $A \subset Y$. It easy to see that $\delta(A+B) \leq \delta(A)+\delta(B)$ for $A, B \in 2^{Y}$, where $A+B=\{a+b: a \in A, b \in B\}$. For $F: K \rightarrow n(Y)$ we denote by cl $F$ the multifunction defined as $(\operatorname{cl} F)(x)=\operatorname{cl} F(x), x \in K$. A function $f: K \rightarrow Y$ such that $f(x) \in F(x)$ for all $x \in K$ is said to be a selection of the multifunction $F$. We write $a^{0}(x)=x$ for $x \in K$ and $a^{n+1}=a^{n} \circ a$ for $n \in \mathbb{N}_{0}, a: K \rightarrow K$.

Theorem 1. Let $F: K \rightarrow n(Y), \Psi: Y \rightarrow Y, a: K \rightarrow K, \lambda \in(0,1)$,

$$
d(\Psi(x), \Psi(y)) \leq \lambda d(x, y) \quad \text { for } x, y \in Y
$$

and

$$
\sup \{\delta(F(x)): x \in K\}<\infty .
$$

(1) If $Y$ is complete and

$$
\Psi(F(a(x))) \subset F(x), \quad x \in K,
$$

then there exists a unique selection $f$ of the multifunction $\mathrm{cl} F$ such that $\Psi \circ f \circ a=f$.

(2) If

$$
F(x) \subset \Psi(F(a(x))), \quad x \in K,
$$

then $F$ is a single-valued function and $\Psi \circ F \circ a=F$.

Proof. (1) Let $x \in K$. Replacing $x$ by $a^{n}(x)$ in (4) we get

$$
\Psi\left(F\left(a^{n+1}(x)\right)\right) \subset F\left(a^{n}(x)\right)
$$


for all $n \in \mathbb{N}_{0}$. Hence

$$
\Psi^{n+1}\left(F\left(a^{n+1}(x)\right)\right) \subset \Psi^{n}\left(F\left(a^{n}(x)\right)\right), \quad n \in \mathbb{N}_{0}
$$

and $\left(\operatorname{cl} \Psi^{n}\left(F\left(a^{n}(x)\right)\right)\right)_{n \in \mathbb{N}_{0}}$ is a decreasing sequence of closed sets in a Banach space. Moreover, in virtue of (3),

$$
\delta\left(\operatorname{cl} \Psi^{n}\left(F\left(a^{n}(x)\right)\right)\right) \leq \lambda^{n} \delta\left(F\left(a^{n}(x)\right)\right),
$$

so $\lim _{n \rightarrow \infty} \delta\left(\operatorname{cl} \Psi^{n}\left(F\left(a^{n}(x)\right)\right)\right)=0$. Therefore

$$
\lim _{n \rightarrow \infty} \operatorname{cl} \Psi^{n}\left(F\left(a^{n}(x)\right)\right)=\bigcap_{n \in \mathbb{N}_{0}} \operatorname{cl} \Psi^{n}\left(F\left(a^{n}(x)\right)\right)=: f(x)
$$

is singleton. Of course $f(x) \in \operatorname{cl} F(x)$ and as $\Psi$ is continuous

$$
\begin{aligned}
\Psi(f(a(x))) & =\Psi\left(\lim _{n \rightarrow \infty} \operatorname{cl} \Psi^{n}\left(F\left(a^{n}(a(x))\right)\right) \subset \lim _{n \rightarrow \infty} \operatorname{cl} \Psi^{n+1}\left(F\left(a^{n+1}(x)\right)\right)\right. \\
& =f(x),
\end{aligned}
$$

so $\Psi \circ f \circ a=f$.

It remains to show the uniqueness of $f$. Suppose that $f, g$ are selections of cl $F$ and $\Psi \circ f \circ a=f, \Psi \circ g \circ a=g$. By induction we obtain that $\Psi^{n} \circ f \circ a^{n}=f$ and $\Psi^{n} \circ g \circ a^{n}=g$ for $n \in \mathbb{N}_{0}$. Hence, for $x \in K$,

$$
\begin{aligned}
d(f(x), g(x)) & =d\left(\Psi^{n} \circ f \circ a^{n}(x), \Psi^{n} \circ g \circ a^{n}(x)\right) \\
& \leq \lambda^{n} d\left(f\left(a^{n}(x)\right), g\left(a^{n}(x)\right)\right) \leq \lambda^{n} \delta\left(F\left(a^{n}(x)\right)\right) .
\end{aligned}
$$

Since $\lim _{n \rightarrow \infty} \lambda^{n} \delta\left(F\left(a^{n}(x)\right)\right)=0$, we have $f=g$.

(2) By (5) we obtain

$$
F(x) \subset \Psi^{n}\left(F\left(a^{n}(x)\right)\right) \subset \Psi^{n+1}\left(F\left(a^{n+1}(x)\right)\right), \quad n \in \mathbb{N}_{0}, x \in K .
$$

So $\left(\Psi^{n}\left(F\left(a^{n}(x)\right)\right)\right)_{n \in \mathbb{N}_{0}}$ is an increasing sequence of sets in a normed space with the diameter

$$
\delta\left(\Psi^{n}\left(F\left(a^{n}(x)\right)\right)\right) \leq \lambda^{n} \delta\left(F\left(a^{n}(x)\right)\right),
$$

which converges to 0 as $n \rightarrow \infty$. Therefore $\Psi^{n} \circ F \circ a^{n}(x)$ is single-valued for all $n \in \mathbb{N}_{0}, x \in K$ and $\Psi \circ F \circ a=F$.

Example. Let $K=Y=\mathbb{R}, f: \mathbb{R} \rightarrow \mathbb{R}$ be a linear function, $\Psi(x)=\frac{1}{2} x, a(x)=$ $2 x$ and $F(x)=[f(x), f(x)+c]$, where $c \in \mathbb{R}_{+} \cdot$ Then

$$
\Psi(F(a(x)))=\left[f(x), f(x)+\frac{c}{2}\right] \subset F(x)
$$

and

$$
\Psi^{n}\left(F\left(a^{n}(x)\right)\right)=\left[f(x), f(x)+\frac{c}{2^{n}}\right]
$$

converges to $f(x)$. 
Theorem 2. Assume that $Y$ is complete, $F, G: K \rightarrow b d(Y), 0 \in G(x)$ for all $x \in K, \Psi: Y \rightarrow Y, a: K \rightarrow K, \lambda \in(0,1)$,

$$
\begin{aligned}
& d(\Psi(x), \Psi(y)) \leq \lambda d(x, y) \quad \text { for } x, y \in Y, \\
& M:=\sup \{\delta(F(x)+G(x)): x \in K\}<\infty
\end{aligned}
$$

and

$$
\Psi(F(a(x))) \subset F(x)+G(x), \quad x \in K .
$$

Then there exists a unique function $f$ such that $\Psi \circ f \circ a=f$ and

$$
d(f(x), F(x)) \leq \frac{1}{1-\lambda} M, \quad x \in K .
$$

Proof. Let $x \in K$. Replacing $x$ by $a^{n}(x)$ in (6) we obtain

$$
\Psi\left(F\left(a^{n+1}(x)\right)\right) \subset F\left(a^{n}(x)\right)+G\left(a^{n}(x)\right)
$$

and, as $0 \in G(x)$, we have

$$
F\left(a^{n}(x)\right) \subset F\left(a^{n}(x)\right)+G\left(a^{n}(x)\right)
$$

for $n \in \mathbb{N}_{0}$. Thus

$$
\begin{aligned}
& h\left(\Psi^{n+1}\left(F\left(a^{n+1}(x)\right)\right), \Psi^{n}\left(F\left(a^{n}(x)\right)\right)\right) \\
& \quad \leq \lambda^{n} h\left(\Psi\left(F\left(a^{n+1}(x)\right)\right), F\left(a^{n}(x)\right)\right) \leq \lambda^{n} \delta\left(F\left(a^{n}(x)\right)+G\left(a^{n}(x)\right)\right)
\end{aligned}
$$

for $n \in \mathbb{N}_{0}$. Hence, for $k \in \mathbb{N}, n \in \mathbb{N}_{0}$, we obtain

$$
\begin{aligned}
h & \left.\Psi^{n+k}\left(F\left(a^{n+k}(x)\right)\right), \Psi^{n}\left(F\left(a^{n}(x)\right)\right)\right) \\
& \leq \sum_{i=0}^{k-1} \lambda^{n+i} \delta\left(F\left(a^{n+i}(x)\right)+G\left(a^{n+i}(x)\right)\right) \\
& =\sum_{j=n}^{n+k-1} \lambda^{j} \delta\left(F\left(a^{j}(x)\right)+G\left(a^{j}(x)\right)\right) \leq \sum_{j=n}^{n+k-1} \lambda^{j} M .
\end{aligned}
$$

Since $\lim _{n \rightarrow \infty} \sum_{j=n}^{n+k-1} \lambda^{j} M=0$, the sequence $\left(\Psi^{n}\left(F\left(a^{n}(x)\right)\right)\right)_{n \in \mathbb{N}_{0}}$ is a Cauchy sequence. As $(b c l(Y), h)$ is a complete metric space, there exists the limit $\lim _{n \rightarrow \infty} \mathrm{cl} \Psi^{n}\left(F\left(a^{n}(x)\right)\right)$. Moreover, the diameter

$$
\delta\left(\operatorname{cl} \Psi^{n}\left(F\left(a^{n}(x)\right)\right)\right) \leq \lambda^{n} \delta\left(F\left(a^{n}(x)\right)\right)
$$

is convergent to 0 as $n \rightarrow \infty$. Therefore

$$
\lim _{n \rightarrow \infty} \operatorname{cl} \Psi^{n}\left(F\left(a^{n}(x)\right)\right)=: f(x)
$$

is singleton and as $\Psi$ is continuous

$$
\begin{aligned}
\Psi(f(a(x))) & =\Psi\left(\lim _{n \rightarrow \infty} \mathrm{cl} \Psi^{n}\left(F\left(a^{n}(a(x))\right)\right) \subset \lim _{n \rightarrow \infty} \mathrm{cl} \Psi^{n+1}\left(F\left(a^{n+1}(x)\right)\right)\right. \\
& =f(x) .
\end{aligned}
$$


In this way we have shown that $\Psi \circ f \circ a=f$. By (7), with $n=0$, we have

$$
h\left(\operatorname{cl} \Psi^{k}\left(F\left(a^{k}(x)\right)\right), \operatorname{cl} F(x)\right) \leq \sum_{j=0}^{k-1} \lambda^{j} M .
$$

Consequently

$$
d(f(x), F(x)) \leq \sum_{j=0}^{\infty} \lambda^{j} M=\frac{1}{1-\lambda} M
$$

and

$$
f(x) \in F(x)+\frac{1}{1-\lambda} M S,
$$

where $S$ is a closed unit ball.

It remains to prove the uniqueness of $f$. Let $f, g$ be such that $\Psi \circ f \circ a=$ $f, \Psi \circ g \circ a=g$ and $f(x), g(x) \in F(x)+\frac{1}{1-\lambda} M S$ for $x \in K$. By induction we get $\Psi^{n} \circ f \circ a^{n}=f$ and $\Psi^{n} \circ g \circ a^{n}=g$ for $n \in \mathbb{N}_{0}$. Hence

$$
\begin{aligned}
d(f(x), g(x)) & =d\left(\Psi^{n} \circ f \circ a^{n}(x), \Psi^{n} \circ g \circ a^{n}(x)\right) \\
& \leq \lambda^{n} d\left(f\left(a^{n}(x)\right), g\left(a^{n}(x)\right)\right) \\
& \leq \lambda^{n} \delta\left(F\left(a^{n}(x)\right)+\frac{1}{1-\lambda} M S\right), \quad n \in \mathbb{N} .
\end{aligned}
$$

Since $\lim _{n \rightarrow \infty} \lambda^{n} \delta\left(F\left(a^{n}(x)\right)+\frac{1}{1-\lambda} M S\right)=0$, we have $f=g$.

In a similar way we obtain

Theorem 3. Assume that $Y$ is complete, $F, G: K \rightarrow b d(Y), 0 \in G(x)$ for all $x \in K, \Psi: Y \rightarrow Y, a: K \rightarrow K, \lambda \in(0,1)$,

$$
\begin{gathered}
d(\Psi(x), \Psi(y)) \leq \lambda d(x, y) \quad \text { for } x, y \in Y, \\
M:=\sup \{\delta(\Psi(F(a(x)))+G(x)): x \in K\}<\infty
\end{gathered}
$$

and

$$
F(x) \subset \Psi(F(a(x)))+G(x), \quad x \in K .
$$

Then there exists a unique function $f$ such that $\Psi \circ f \circ a=f$ and

$$
d(f(x), F(x)) \leq \frac{1}{1-\lambda} M, \quad x \in K .
$$


Example. Let $K=Y=\mathbb{R}, f: \mathbb{R} \rightarrow \mathbb{R}$ be a linear function, $\Psi(x)=\frac{1}{2} x, a(x)=$ $2 x$ and $F(x)=[f(x)+\sin x, f(x)+c+\sin x]$, where $c \in \mathbb{R}_{+}$. Then

$$
\begin{gathered}
\Psi(F(a(x)))=\left[f(x)+\frac{1}{2} \sin (2 x), f(x)+\frac{c}{2}+\frac{1}{2} \sin (2 x)\right] \\
\subset F(x)+\left[-\sin x-\frac{1}{2}, \frac{3}{2}\right], \\
\sup \left\{\delta\left(F(x)+\left[-\sin x-\frac{1}{2}, \frac{3}{2}\right]\right): x \in \mathbb{R}\right\} \leq c+3
\end{gathered}
$$

and

$$
\Psi^{n}\left(F\left(a^{n}(x)\right)\right)=\left[f(x)+\frac{1}{2^{n}} \sin \left(2^{n} x\right), f(x)+\frac{c}{2^{n}}+\frac{1}{2^{n}} \sin \left(2^{n} x\right)\right]
$$

converges to $f(x)$.

Corollary 4. Let $Y$ be complete, $F: K \rightarrow b d(Y), \Psi: Y \rightarrow Y, a: K \rightarrow K, N \in$ $\mathbb{R}, \lambda \in(0,1)$,

$$
\begin{gathered}
d(\Psi(x), \Psi(y)) \leq \lambda d(x, y) \quad \text { for } x, y \in Y, \\
M:=\sup \{\delta(F(x)): x \in K\}<\infty
\end{gathered}
$$

and

$$
h(\Psi(F(a(x))), F(x)) \leq N, \quad x \in K .
$$

Then there exists a unique function $f$ such that $\Psi \circ f \circ a=f$ and

$$
d(f(x), F(x)) \leq \frac{1}{1-\lambda}(M+2 N), \quad x \in K .
$$

Proof. By (8) we have

$$
\Psi(F(a(x))) \subset F(x)+N S, \quad F(x) \subset \Psi(F(a(x)))+N S, \quad x \in K,
$$

where $S$ is a closed unit ball. Moreover,

$$
\sup \{\delta(F(x)+N S): x \in K\} \leq M+2 N,
$$

whence by Theorem 2 or 3 we get the assertion.

We end the paper with a result on the stability of the equation $\Psi \circ f \circ a=f$. Theorem 5. Assume that $Y$ is complete, $f: K \rightarrow Y, \Psi: Y \rightarrow Y, a: K \rightarrow$ $K, k: K \rightarrow[0, \infty)$ is bounded, $\lambda \in(0,1)$,

$$
d(\Psi(x), \Psi(y)) \leq \lambda d(x, y) \quad \text { for } x, y \in Y
$$

and

$$
\|\Psi(f(a(x)))-f(x)\| \leq k(x) \quad \text { for } x \in K .
$$


Then there exists a unique function $g$ such that $\Psi \circ g \circ a=g$ and

$$
\|f(x)-g(x)\| \leq \frac{2}{1-\lambda} M, \quad x \in K,
$$

where $M=\sup \{|k(x)|: x \in K\}$.

Proof. Let $F(x):=\{f(x)\}$. Then

$$
\Psi(F(a(x))) \subset F(x)+k(x) S
$$

and

$$
\sup \{\delta(F(x)+k(x) S): x \in K\} \leq 2 M .
$$

By Theorem 2 there exists a unique function $g$ such that $\Psi \circ g \circ a=g$ and $\|f(x)-g(x)\| \leq \frac{2}{1-\lambda} M$ for $x \in K$. The assertion follows from Theorem 3 or Corollary 4.

As it was observed by Forti [5] and explicitly proved in [2], from stability results concerning the equation $\Psi \circ f \circ a=f$ we can easily derive the stability of functional equations in several variables, for example: the Cauchy equation $f(x+y)=f(x)+f(y)$, the Jensen equation $f\left(\frac{x+y}{2}\right)=\frac{f(x)+f(y)}{2}$ or the quadratic equation $f(x+y)+f(x-y)=2 f(x)+2 f(y)$.

The results that we have been obtained in this paper correspond to the result in [3] and complement them.

Open Access. This article is distributed under the terms of the Creative Commons Attribution License which permits any use, distribution, and reproduction in any medium, provided the original author(s) and the source are credited.

\section{References}

[1] Aczél, J.: Lectures on Functional Equations and Their Applications. Academic Press, New York (1966)

[2] Brzdȩk, J.: On a method of proving the Hyers-Ulam stability of functional equations on restricted domains. AJMAA 6, 1-10 (2009)

[3] Brzdȩk, J., Popa, D., Xu, B.: Selections of set-valued maps satisfying a linear inclusions in single variable via Hyers-Ulam stability. Nonlinear Anal. 74, 324-330 (2011)

[4] Forti, G.L.: Hyers-Ulam stability of functional equations in several variables. Aequ. Math. 50, 143-190 (1995)

[5] Forti, G.L.: Comments on the core of the direct method for proving Hyers-Ulam stability of functional equations. J. Math. Anal. Appl. 295, 127-133 (2004)

[6] Gajda, Z., Ger, R.: Subadditive multifunctions and Hyers-Ulam stability. Numer. Math. 80, 281-291 (1987)

[7] Hyers, D.H.: On the stability of the linear functional equation. Proc. Natl. Acad. Sci. USA 27, 222-224 (1941)

[8] Hyers, D.H., Isac, G., Rassias, Th.M.: Stability of Functional Equations in Several Variables. Birkhäuser, Boston (1998)

[9] Kuczma, M.: An Introduction to the Theory of Functional Equations and Inequalities. Uniwersytet Śląski, Katowice, PWN, Warsaw (1985) 
[10] Nikodem, K., Popa, D.: On selections of general linear inclusions. Publ. Math. Debrecen 75, 239-249 (2009)

[11] Páles, Z.: Generalized stability of the Cauchy functional equation. Aequ. Math. 56, 222-232 (1998)

[12] Páles, Z.: Hyers-Ulam stability of the Cauchy functional equation on square-symmetric grupoids. Publ. Math. Debrecen 58, 651-666 (2001)

[13] Popa, D.: A stability result for a general linear inclusion. Nonlinear Funct. Anal. Appl. 3, 405-414 (2004)

[14] Popa, D.: Functional inclusions on square-symmetric grupoids and Hyers-Ulam stability. Math. Inequal. Appl. 7, 419-428 (2004)

[15] Popa, D.: A property of a functional inclusion connected with Hyers-Ulam stability. J. Math. Inequal. 4, 591-598 (2009)

[16] Rassias, Th.M.: On the stability of linear mappings in Banach spaces. Proc. Am. Math. Soc. 72, 297-300 (1978)

[17] Smajdor, W.: Superadditive set-valued functions. Glas. Mat. 21, 343-348 (1986)

Magdalena Piszczek

Institute of Mathematics

Pedagogical University

Podchorążych 2

30-084 Kraków

Poland

e-mail: magdap@up.krakow.pl

Received: January 2, 2012

Revised: January 21, 2012 